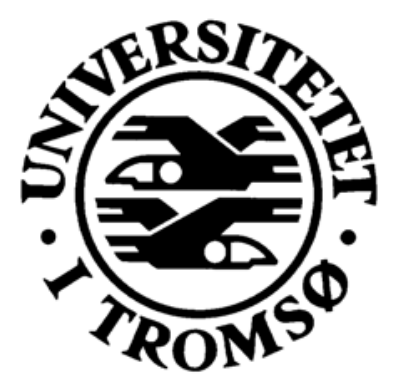

\title{
Contingent payments in selection contests
}

\author{
by
}

Christian Riis and Derek J. Clark

Working Paper Series in Economics and Management No. 03/05, April 2005

\section{Department of Economics and Management \\ Norwegian College of Fishery Science \\ University of Tromsø \\ Norway}




\title{
Contingent payments in selection contests
}

\author{
by \\ Christian Riis and Derek J. Clark
}

\begin{abstract}
Author affiliation:
Derek J. Clark: Department of Economics and Management, NFH, University of Tromsø, N-9037 Tromsø, Norway, and Bodø Graduate School of Business, N-8049 Bodø, Norway. Email: Derek.Clark@nfh.uit.no

Christian Riis: Department of Economics, Norwegian School of Management, P.O.Box 580, N-1302 Sandvika, Norway, and Department of Economics and Management, NFH, University of Tromsø, N-9037 Tromsø, Norway. Email: christian.riis@bi.no
\end{abstract}




\begin{abstract}
The early literature on research contests stressed the advantages of a fixed prize in inspiring R\&D effort. More recently the focus has moved towards endogenizing the rewards to research activity in these tournament settings, since this can induce extra effort or enhance the surplus of the buyer. We focus on a research contest as a means of selecting a partner for an R\&D enterprise, in an informational setting in which the established providers of R\&D services know more about each others' relative capabilities than does the buyer/sponsor. This asymmetry creates a source of inefficiency if a rank order contest is used as a selection device; we show how the contest can be modified to improve selection efficiency, while maintaining its simplicity (as only ordinal information is required). The modification that we suggest involves endogenizing the prizes that are awarded contingent upon whether a contestant wins or loses the contest. Furthermore, the payment system and the selection mechanism are detail free.
\end{abstract}

Keywords: research contest, selection, rank-order, contingent payment.

JEL Classification: D82, M52, D72 


\section{Introduction}

In the funding of commercial research and development (R\&D) projects, payment structures have long been used that comprise a fixed fee and a payment contingent upon some pre-specified realization or event. Windus and Schiffel (1976) present a summary of funding practice by public bodies in several countries in which a common element is a fixed level of funding, and a variable component comprising a proportion of the profit that the developing firm can keep in the event of a commercially viable innovation. Early models of R\&D contests involved a sponsor offering a fixed prize for an innovation (Taylor, 1995, and Fullerton and McAfee, 1999). Recently there has been a move away from fixed-prize research contests, as advocated by Che and Gale (2003), who give the following examples of contests in which endogenous prizes have been used: (i) in the procurement of a high-speed train system in Korea, firms proposed designs and prices for these designs, (ii) grant competitions sponsored by the National Science Foundation, (iii) defense contractors often use R\&D investments to produce prototypes, and then bid for the final production contract.

The focus of this paper is how a simple endogenous prize research contest can be used by a buyer to select a partner for a $R \& D$ enterprise when there is asymmetric information. To be specific, consider a research contest in which a firm is seeking a partner in the development and subsequent marketing of a new product. Selecting the correct partner for this venture would be an obvious aim here, but the established providers of R\&D services are likely to know more about each others' capabilities than a potential sponsor. Hence a contest may be instigated to gauge the suitability of different research laboratories for this task. Our analysis presents a simple, detail-free method for selecting the most suitable partner by setting up the correct menu of rewards in a simple rank-order contract. A high quality partner is identified as requiring a larger fee (share of the profits) if it is successful in developing the new product, but will be willing to bear a larger proportion of the cost if unsuccessful than would a less able competitor. Such costsharing arrangements in R\&D have long been in existence in several countries (see Keck, 
1988). Fullerton et al. (2002) note that one benefit with a more flexible prize schedule is that the contest designer is then relieved of the burden of calculating the correct level of the fixed prize. ${ }^{1}$ In the context of military acquisitions, Fullerton (1995) has suggested that a contractor may submit a proposal that incorporates some element of an appropriate reward for his efforts; our analysis gives a formal basis for this proposal.

Our work builds upon that of Che and Gale (2003) and Fullerton et al. (2002) who have analyzed models of research contests in which the prize for winning is set by means of an auction mechanism. Firms compete in terms of the quality of their innovation and the price that they require for selling it, and the buyer's aim in both of these models is to maximize his surplus. The role of contests as an incentive device has been widely analyzed in the literature, especially relating to the internal labour market. ${ }^{2}$ In this paper, we concentrate on a contest as a selection device, in which the aim is to identify the best individual, firm, or research team for a specific task. Indeed in much of the contest and tournament literature selection has not been an issue since participants are assumed to be symmetric ex ante so that the contest designer is indifferent as to the identity of the agent that wins the contest. However, allocating the contest prize to the most suitable participant is an issue that arises in research contests, internal labour markets, and other applications. $^{3}$

A selection problem arises in a contest if the participants have different ability potential. Heterogeneity has been shown to disturb the contest as an incentive device, and may also

\footnotetext{
${ }^{1}$ Judd et al. (2003) consider a patent race in which the patent granting authority can choose when to grant the patent and the size of the reward (through instruments such as patent length and breadth). Our mechanism would relieve the authority of the second task.

${ }^{2}$ For example, Lazear and Rosen (1981), Nalebuff and Stiglitz (1983), and Green and Stokey (1983); see also McLaughlin (1988), for an overview. Also later work on contest design, has had the maximization of revenue (or effort) as a goal: Moldovanu and Sela (2002) analyze contests in which the aim is to maximize total effort, or to maximize the highest individual effort, and Singh and Wittman (2001) consider a contest designer whose goal is the maximization of expected revenue.

${ }^{3}$ Gifford and Kenney (1986) represent an early attempt to highlight the role of contests in matching the correct worker to the correct task. They state that "Considerable information is needed for the correct placement of individuals in various labor market positions" (Gifford and Kenney, 1986; 305). We show that this is not necessarily the case. Employee selection is also briefly touched upon in Lazear and Rosen (1981) and in O'Keeffe, Viscusi and Zeckhauser (1984); see also Meyer (1991).
} 
disturb it as a selection device. ${ }^{4}$ Lazear and Rosen (1981) and O'Keeffe, Viscusi and Zeckhauser (1984) demonstrate that a favourite often has weak incentives to exert effort if he faces an underdog due to the fact that he has good chances to win the contest even at a modest effort level. To reestablish the first best effort incentives, Lazear and Rosen (1981) consider handicapping, as well as a process of self-selection of contestants into different leagues or contests. Handicapping is also used by Che and Gale (2003) in their analysis of optimal research contests when firms are asymmetric and this asymmetry is common knowledge. ${ }^{5}$ Bhattacharya and Guasch (1988) and Yun (1997) consider the self-selection of participants into parallel contests; in the former, workers self-select and the prize received depends upon a comparison with workers from the lowest ability contest (i.e. between cohorts), and the latter employs self-selection of two types in which the prize is awarded purely on the basis of a within-cohort comparison. ${ }^{6}$

The real problem in selection contests arises if the principal is unaware of the potential heterogeneity of the contestants, in which case handicapping is ruled out as a mechanism. Furthermore, if the contestants know more about each other's potential ability than the principal knows, the contest is biased. We believe that this informational asymmetry fits many real life situations well. In a research contest, the providers of R\&D services would know more about each others' relative capabilities than would a potential buyer. This generates an asymmetry, which becomes a source of inefficiency, if a rank order contest is used as a selection device. As an illustration, consider a research contest in which an R\&D provider with high expected ability faces an opponent that it assesses to be of lower ability. In such a situation, the high ability team has an incentive to slack off in the contest. This behavior arises precisely because it expects to meet an inferior opponent; the high ability team thus reduces its effort and its associated cost. If the R\&D team with low expected ability turns out to be more capable than expected, then it benefits from the opponent's tendency to slack off, and adopts a more aggressive stance.

\footnotetext{
4 "The real problem with tournaments with heterogeneous contestants arises if the contestants' types cannot be identified" (McLaughlin, 1988; 248).

${ }^{5}$ Hence an optimal design focuses on effort in the R\&D contest (more specifically the surplus offered to the buyer of the new technology).

${ }^{6}$ The aim of the principal in these papers is to induce first best effort, and the focus is not on selection per se.
} 
As a result, the high ability contestant occasionally loses to inferior opponents. Hence, in instances where the agents are better informed than the principal, there is a risk of selecting an inferior agent. ${ }^{7}$

It follows that running a fair contest (a contest that is symmetric with respect to the permutations of the contestants) does not yield first best selection in the case of heterogeneous contestants. However running a biased contest requires information that is often not available to the principal. In the present paper, we propose an alternative procedure based on endogenous prizes. The principal only has access to an ordinal comparison of the contest variable, but can make the reward contingent upon winning or losing. The main conclusion is that the principal can improve selection efficiency by letting each contestant trade off a higher winning prize at the cost of a reduction in the losing prize. ${ }^{8}$ The intuition is that a contestant who faces an opponent that he expects to be of low ability, is willing to accept a large reduction in the losing prize for a given increase in the winning prize, simply because his overall winning probability is higher. On the other hand, a contestant who faces an opponent he believes to be of high ability is better off choosing a smaller gap. Consequently, giving each contestant the option of increasing his winning prize at the cost of a reduction in the losing prize, affects his stake in the contest, and hence his willingness to compete fiercely. This counteracts the selection bias described above; in our modified contest the bidding incentives of a high ability contestant are reinforced since he has more to win. ${ }^{9}$

In Clark and Riis (2001), we analysed how a bonus system can be used in order to reduce the risk of selecting the inferior agent in a contest. That is, by carrying out ordinal comparisons of the winner's effort with certain test standards, and rewarding the winner

\footnotetext{
${ }^{7}$ This bias is acknowledged in the literature; the result is shown by for instance Amann and Leininger (1996).

${ }^{8}$ The payment structure that we suggest is simple and detail-free.

${ }^{9}$ O'Keeffe, Viscusi and Zeckhauser (1984) examine a related incentive mechanism in their discussion of self-sorting into leagues of high ability versus low ability. They point out that the "climbing-incentives", which means that low ability types infiltrate "high -ability contests", can be dampened by increasing the prize spread in the high ability contest (obviously, low ability types receive the loser prize more frequently). Another example is given by Landers et al. (1996) who focus on promotion decisions in law firms where there is an incentive to promote associates who have a propensity to work very hard. "Rat race" equilibrium occurs since the principal may use work hours as a selection device. By properly designing "wage-hours" packages, the principal may separate workers with low and high disutility of work.
} 
adequately if these are passed, we demonstrated that the bias in favour of agents with low expected capability disappears. First-best selection is achieved, albeit under a restricted set of probability distributions: it is assumed that abilities are drawn from two uniform distributions that are identical up to a location shift. The model that we present in this paper is a significant generalization of this work. ${ }^{10}$

The paper is organized as follows. Section 2 presents the basic model. Section 3 recaps the selection bias that occurs in a fixed prize contest, while Section 4 presents a prize structure that implements perfect selection. Brief conclusions are offered in Section 5.

\section{The model.}

Consider a two-player contest, in which the contestant who submits the largest bid wins, and all bids are forfeited. The two contestants are recruited from two populations with identical ability distributions $F(v)$ defined on $[0, \bar{v}]$, where possibly $\bar{v}=\infty$. Assume that in the process of recruiting a screening occurs that guarantees that the contestant at least passes a certain threshold. To be concrete, assume the single contestant is randomly drawn from a truncated distribution $F_{i}(v):=F(v) \mid v \geq \underline{v}_{i}$. The truncation levels result from some stochastic process, and we denote by $\underline{v}_{H}$ and $\underline{v}_{L}$ the highest and lowest realized truncation points. We need not further specify the exact nature of the determination of these cut-off points for our results to hold, and we do not consider the case in which they are equal since our focus is on selection in asymmetric contests (if the cut-off points are equal, selection efficiency is not an issue as the most able contestant typically wins the contest). Hence the contestant with ability drawn from the $F_{H}(v)$ distribution is expectedly superior. Each contestant knows the screening efficiency (i.e. the distribution of ability) of the opponent, however the principal is unaware of which group each contestant is recruited from; in addition, the principal does not know the thresholds $\underline{v}_{H}$ and $\underline{v}_{L}$. Indeed, the aim of the paper is to develop a contest mechanism that ensures selection efficiency independent of the truncation levels $\underline{v}_{H}$ and $\underline{v}_{L}$. Hence from the

\footnotetext{
${ }^{10}$ Another paper that looks at pure selection in contests is Hvide and Kristiansen (2003). Their model is different to those discussed above since the strategies of the participants relate to risk taking rather than effort level.
} 
principal's point of view the contest is symmetric; however he is aware of the fact that the contest is asymmetric from the contestants' point of view, unless the thresholds $\underline{v}_{H}$ and $\underline{v}_{L}$ are identical. The problem for the principal is to design a contest such that the winner is the higher ability participant.

The cost of making a bid $x$ is denoted $c(x, v)$. We assume that $c(x, v)$ is continuous with continuous first-order derivatives, where $c_{v}(x, v)<0, c_{x}(x, v)>\varepsilon>0$ and $c_{v x}(x, v)<0$, and that $c(0, v)=0$. Hence the marginal cost, as well as the total cost of making a bid decreases in ability $v$. It is assumed that the two contestants cannot directly report to or inform the principal about the differences in the shapes of the two $F_{i}$-functions, or their true $\operatorname{cost} c(x, v)$. Information can only be transmitted indirectly in the form of contest effort $^{11}$, where we assume that the principal obtains ordinal information only. If the degree of asymmetry, the ability or the cost functions are communicable (by an ordinal or cardinal measure) a much larger set of designs are feasible than the contest considered here.

The contestant who makes the largest bid $x$ wins and receives a prize $W$, whereas the loser receives a prize $w$. The difference $m:=W$-w denotes the prize premium. In the case of a draw, each contestant wins with a probability equal to 0.5 .

Since the aim of the principal is to select the agent of highest ability, it is instructive to first characterize the selection inefficiency that occurs in the fixed prize contest, where $W$ and $w$ are exogenously given (Section 3). Then we will show how a prize menu $m(W)$ can be designed in order to ensure selection efficiency (Section 4). The idea is that each contestant, in addition to making a bid $x$, chooses an individual prize premium from the menu $m(W)$.

\section{The fixed prize contest.}

In the fixed prize contest, the participant that submits the highest bid wins a prize of value $W$ while the loser receives $w$. $W$ and $w$ are given numbers. Denote by type $H$, a 
contestant with ability drawn from the $F_{H}$ distribution, the distribution that has most weight in the upper tail, and as his opponent, type L. $v_{H}$ and $v_{L}$ denote realizations of the two contestants' abilities; the realizations are private information.

Equilibrium in the contest is characterized by two strictly increasing continuous bid functions, $x_{i}=b_{i}\left(v_{i}\right), i=H, L$. Hence type $\mathrm{H}$, if he submits a bid $x_{H}$ beats all types $\mathrm{L}$ of ability $v$ below $v_{L}^{c}$, where $v_{L}^{c}$ is determined by the inverse equilibrium bid function $v_{L}^{c}=$ $b_{L}^{-1}\left(x_{H}\right)$. We can therefore write type H's expected payoff as a function of the set of type $\mathrm{L}$ abilities that he beats ${ }^{12}$ and his own ability $v_{H}$,

$$
\begin{aligned}
\pi_{H} & =\operatorname{Pr}\left(x_{H}>x_{L}\right) W+\left(1-\operatorname{Pr}\left(x_{H}>x_{L}\right)\right) w-c\left(x_{H}, v_{H}\right) \\
& =W-\left(1-\operatorname{Pr}\left(x_{H}>x_{L}\right)\right) m-c\left(x_{H}, v_{H}\right) \\
& =W-\left(1-F_{L}\left(v_{L}{ }^{c}\right)\right) m-c\left(b_{L}\left(v_{L}{ }^{c}\right), v_{H}\right) \\
& =\pi_{H}\left(v_{L}^{c} ; v_{H}\right)
\end{aligned}
$$

Hence, the optimal bid is implicitly determined by the critical $v_{L}^{c}$ value which maximizes $\pi_{H}$. Differentiating $\pi_{H}$ with respect to $v_{L}^{c}$ yields the first order condition:

$$
\frac{\partial \pi_{H}}{\partial v_{L}^{c}}=f_{L}\left(v_{L}^{c}\right) m-c_{x}\left(b_{L}\left(v_{L}^{c}\right), v_{H}\right) b_{L}^{\prime}\left(v_{L}^{c}\right)=0,
$$

where a prime denotes the first order derivative. Denote by $e\left(v_{H}\right)$, and its inverse $e^{-1}\left(v_{L}^{c}\right)$, the equilibrium correspondence functions implicitly defined by $b_{L}(e) \equiv b_{H}(v)$. Replacing $v_{H}$ by $e^{-1}\left(v_{L}^{c}\right)$ in type H's first order condition (2), and $v_{L}^{c}$ by $e\left(v_{H}\right)$ in the corresponding expression for type L, yields a system of two differential equations

$$
b_{L}^{\prime}(v)=\frac{f_{L}(v) m}{c_{x}\left(b_{L}(v), e^{-1}(v)\right)} \equiv K_{L}\left(b_{L}, b_{H}, v\right)
$$

$$
b_{H}^{\prime}(v)=\frac{f_{H}(v) m}{c_{x}\left(b_{H}(v), e(v)\right)} \equiv K_{H}\left(b_{H}, b_{L}, v\right)
$$

\footnotetext{
${ }^{11}$ Our mechanism is partly inspired by the "Wilson Doctrine" in auction theory, i.e. the focus on detail-free auction rules that are independent of models specifics such as functional forms or distributions of signals, see Dasgupta and Maskin (1998).

${ }^{12}$ The probability of identical bids is zero in equilibrium, to simplify the expressions this event is neglected.
} 
As shown in the Appendix, from the real theory of differential equations it follows that a unique solution $\left[b_{H}(v), b_{L}(v)\right]$ on $[0, \bar{v}]$ of the system (3) exists, where the equilibrium bidding functions are strictly increasing above zero, have a common ending point $\bar{x}$ and where at most one agent bids zero with a strictly positive probability.

Proposition 1 indicates the selection inefficiency in the fixed prize contest, by showing that the equilibrium bid function for type L lies everywhere above that of the expectedly superior opponent.

Proposition 1: For all $v<\bar{v}$ then $b_{L}(v)>b_{H}(v)$.

\section{Proof:}

If $b_{H}(v)=b_{L}(v)$, then it follows from (3) that $b_{H}^{\prime}(v)>b_{L}^{\prime}(v)$. Hence $b_{H}(v)$ and $b_{L}(v)$ intersect at most once. As $b_{H}(\bar{v})=b_{L}(\bar{v})$, the proposition follows.

\section{The endogenous prize contest}

If units of ability were measurable and comparable, one might consider establishing a direct mechanism in which the contestants truthfully reported their types on a cardinal scale. Based on these reports, the contest could be modified such that a first-best selection was implemented. However, a direct mechanism requires considerably more information than a contest (in addition, qualitatively different information is demanded, as reports have to be given on a cardinal scale). An attractive feature of rank order contests is that the required amount of information required is limited; decisions are made on the basis of ordinal information only.

There are restrictions that limit the set of feasible mechanisms; the principal receives only ordinal information on bids, and he does not know which contestant, if any, is of higher expected ability. Hence the distribution of prizes can only be conditioned on whether a contestant wins or loses. Therefore, we modify the contest by introducing the choice of prize structure as part of the contest. To make the model as simple as possible, we assume the two contestants simultaneously submit, 1) a bid $x$ and 2) a choice of prize premium from a menu $m(W)$. Certainly, this can be interpreted as a sequential game, where the 
contestants choose prize premiums before the contest begins, but where each contestant's choice is not revealed to the opponent.

Assume that the contestants can choose a prize premium from a strictly convex menu $m(W)$. Clearly a higher winning prize must be at the cost of a reduced losing prize, hence $m^{\prime}(W)>1$. The contestants simultaneously choose winning prizes $W_{H}$ and $W_{L}$ and submit bids $x_{H}$ and $x_{L}$. By bidding $x_{H} \mathrm{H}$ beats all L-types with ability below $v_{L}^{c}$, where $v_{L}^{c}$ $=b_{L}^{-1}\left(x_{H}\right)$. Consider type H's expected utility:

$$
\pi_{H}\left(v_{L}^{c}, W_{H} ; v_{H}\right)=W_{H}-\left(1-F_{L}\left(v_{L}^{c}\right)\right) m\left(W_{H}\right)-c\left(b_{L}\left(v_{L}^{c}\right), v_{H}\right) \text {. }
$$

Differentiating $\pi_{H}$ with respect to $W_{H}$ and $v_{L}^{c}$ gives the first order conditions:

$$
\begin{aligned}
& \text { (5a) } \frac{\partial \pi_{H}}{\partial v_{L}}=f_{L}\left(v_{L}^{c}\right) m\left(W_{H}\right)-c_{x}^{\prime}\left(b_{L}\left(v_{L}^{c}\right), v_{H}\right) b_{L}^{\prime}\left(v_{L}^{c}\right)=0 \\
& \text { (5b) } \frac{\partial \pi_{H}}{\partial W_{H}}=1-\left(1-F_{L}\left(v_{L}^{c}\right)\right) m^{\prime}\left(W_{H}\right)=0
\end{aligned}
$$

which yield solutions $W_{H}\left(v_{H}\right)$ and $v_{L}^{c}\left(v_{H}\right)$.

Inserting H's preferred winning prize $W_{i}(v)$ from $(5 \mathrm{~b})$ and the equilibrium correspondence function $e(v)$ into (5a), and doing the same for player $\mathrm{L}$, yields the following system of two differential equations: ${ }^{13}$

$$
b_{H}^{\prime}(v)=\frac{f_{H}(v) m\left(W_{L}(v)\right)}{c_{x}^{\prime}\left(b_{H}(v), e(v)\right)}
$$

(6)

$$
b_{L}^{\prime}(v)=\frac{f_{L}(v) m\left(W_{H}(v)\right)}{c_{x}^{\prime}\left(b_{L}(v), e^{-1}(v)\right)}
$$

Perfect selection efficiency requires that $e(v)=e^{-1}(v)=v$ and $b_{H}(v)=b_{L}(v)=b(v)$. Then (6) implies

$$
f_{H}(v) m\left(W_{L}(v)\right)=f_{L}(v) m\left(W_{H}(v)\right)
$$

\footnotetext{
${ }^{13}$ We have not explicitly given the first-order conditions for type L that lead to the second equation in (6) as they follow the same form as in equation (5).
} 
which has a simple interpretation: perfect selection requires that two contestants of equal abilities have the same bidding incentives at the margin. This means that a type $\mathrm{L}$, of ability $v$, chooses a prize premium $m\left(W_{L}(v)\right)$, which is proportional to the prize premium $m\left(W_{H}(v)\right)$ a type $\mathrm{H}$ of exactly the same ability $v$ would choose, with the relative density $f_{H}(v) / f_{L}(v)$ as a proportionality factor.

We will now demonstrate that there indeed exists a menu $m(W)$ such that the contest yields efficient selection independent of the truncation levels $\underline{v}_{H}$ and $\underline{v}_{L}$. Since it follows from (5b) that $W_{H}$ is strictly increasing in the win probability, $F_{L}\left(v_{L}\right)$, (recall that the $m(W)$ function is assumed to be increasing strictly convex) two contestants with equal win probabilities choose equal prize premiums. That is, $W_{H}(v)=W_{L}\left(h_{k}(v)\right)$ where the function $h_{k}(v)$ is implicitly determined by $F_{L}(v) \equiv F_{H}\left(h_{k}(v)\right)$, where the index $k$ indicates the degree of contest asymmetry, defined by ${ }^{14}$

$$
k:=\frac{1-F\left(\underline{v}_{H}\right)}{1-F\left(\underline{v}_{L}\right)}
$$

Thus $k$ is a number between 0 and 1 , where a low $k$ implies high degree of asymmetry. Since $W_{H}(v)=W_{L}\left(h_{k}(v)\right)$ and $f_{L}(v) / f_{H}(v) \equiv k$ per definition we can write $(7)$ as follows:

$$
k m\left(W_{L}\left(h_{k}(v)\right)\right)=m\left(W_{L}(v)\right) .
$$

Furthermore from the first order condition (5b), it follows that ${ }^{15}$

$$
k m^{\prime}\left(W_{L}\left(h_{k}(v)\right)\right)=m^{\prime}\left(W_{L}(v)\right) .
$$

Combining (8) and (9) yields,

$$
\frac{m^{\prime}\left(W_{L}\left(h_{k}(v)\right)\right)}{m\left(W_{L}\left(h_{k}(v)\right)\right)}=\frac{m^{\prime}\left(W_{L}(v)\right)}{m\left(W_{L}(v)\right)} .
$$

\footnotetext{
${ }^{14}$ Observe there is a simple relationship between $h_{k}(v)$ and $k$, since $F\left(h_{k}(v)\right)=k F(v)+1-k$.

${ }^{15} m^{\prime}\left(W_{H}(v)\right)=m^{\prime}\left(W_{L}\left(h_{k}(v)\right)\right)=\frac{1}{1-F_{L}(v)}=\frac{1}{k\left[1-F_{H}(v)\right]}=\frac{1}{k} m^{\prime}\left(W_{L}(v)\right)$
} 
since $h_{k}(v)$ runs from $v$ to $\infty$ as $k$ goes from 1 to 0 , it follows that the menu $m(W)$ must satisfy

$$
\frac{m^{\prime}(z)}{m(z)}=C_{1} \text { for all z }
$$

which gives the solution

$$
m(W)=C_{0} e^{C_{1} W}
$$

where $C_{l}$ and $C_{0}$ are positive constants. The main result can now be summarized as follows:

\section{Proposition 2}

Perfect selection occurs if contestants simultaneously select contest effort $x$ and winning prize $W$, where the latter choice is made from a menu $m(W)=C_{0} e^{C_{1} W}$ where $C_{0}$ and $C_{1}$ are positive constants.

Inserting $m(W)=C_{0} e^{C_{1} W}$ in the first order condition (5) yields the following differential equation

$$
b^{\prime}(v)=\frac{f(v)}{(1-F(v)) c_{x}^{\prime}(b(v), v) C_{1}}
$$

with boundary condition $b\left(\underline{v}_{H}\right)=0$. Observe that all L-types of ability $v \leq \underline{v}_{H}$ exert zero effort in equilibrium, and hence so does the H-type of ability $\underline{v}_{H}$.

The point here is that a contestant who considers his competitor to be an "underdog", is willing to accept a small increase in the prize received as a winner as compensation for a given reduction in the losing prize $w$, simply because his winning probability is large. Consequently, giving each contestant the option of increasing the winning prize at the cost of decreasing the losing prize affects the bidding incentives. This counteracts the incentive bias that typically occurs in asymmetric contests, where the bidding incentives of "top-dogs" are poor due to the high winning probability. In our modified contest the 
bidding incentives of an expected high ability player are reinforced since he has more to win. Furthermore, the prize menu and the selection mechanism are detail-free.

\section{Conclusion}

The main theoretical contribution of this paper is that a principal can improve selection efficiency in a contest by allowing the contestants to influence the remuneration scheme. The solution that we have presented is simple since it involves only an ordinal comparison of the contest variable, and the reward schedule is independent of the specific details of the models such as the distribution of players' types. Specifically, letting the contestants trade off a higher winning prize at the cost of a lower losing prize achieves the desired result. We have argued that the results may be of use in the design of certain research contests in which a fixed reward cannot achieve all of the sponsor's aims. In our analysis, we have assumed that the contestants' abilities are drawn from two truncated probability distributions, where the underlying distribution is the same for the two contestants. A more general approach would be to specify a larger set of probability functions from which the two specific distributions $F_{L}$ and $F_{H}$ are drawn. In the more general case, perfect selection efficiency is not achievable. Still, an important insight holds, namely that allowing the contestants to increase their stakes in the contest tends to reduce the likelihood of selection inefficiency. The bidding incentives for an expectedly high ability player are reinforced, hence the chance that he loses against inferior opponents decreases. Even though the fine-tuning that is achievable in the case with a common underlying distribution cannot be realized in the general case, selection efficiency is typically improved compared to the fixed prize contest; see the discussion in Riis (2003).

The specific example that we have used to motivate our results has been related to the cofunding of R\&D enterprise, but it should be clear that our results could equally as well be applied to an internal labour markets. Another application of our analysis relates to environmental damage as described by Liston-Heyes (2001) who considers a (full information) contest between a firm that wants to produce a product that will damage the environment and an environmental pressure group that wants to stop this development. 
The firm chooses its own level of profit from the proposed project strategically in order to increase its chances of being allowed to proceed with the development. In equilibrium, this is shown to reduce the amount of environmental damage that occurs compared to the fixed prize (profit) case. As an extension of this, one can imagine a situation with two firms that compete for the product development, and that their ability to limit environmental damage is unknown to the regulator. Indeed, it is not unthinkable that the regulator may not even know which of the competitors is expected to have the better ability. Given that the firm of higher ability will have lower marginal cost of "selling" its product to the regulator through marketing and lobbying campaigns, a smart move for the regulator may be to allow an endogenization of the prize structure along the lines that we have suggested. A firm that will be good at limiting environmental damage from the new development can signal this by choosing a high profit level if successful (by, for example, that the regulator allows access to the market at a low price), against a low level if it is not. 


\section{Appendix.}

We first show that equilibrium bidding functions are: I) strictly increasing above zero, II) have a common ending point and III) that at most one firm may bid zero with a strictly positive probability.

I) Assume an interval [v', $\left.v^{\prime \prime}\right]$ exists at which all L types in this interval bid a given amount $z$. Hence, type H's payoff function is discontinuous and jumps upwards at $x_{H}=z$. Consequently, type $\mathrm{H}$ does not make a bid just below $z$. Thus $z$ may not be L's best reply since type L of type $v \in\left[v^{\prime}, v^{\prime \prime}\right]$ may reduce his bid without reducing the winning probability. II) The ending points are common since no firm bids strictly more than the largest potential amount the competitor bids. III) At most one firm may bid zero with a strictly positive probability; otherwise each firm is better off making a small positive bid with certainty than by a zero bid.

Hence, $\bar{x}=b_{H}(\bar{v})=b_{L}(\bar{v})$ and $b_{H}\left(v_{H}^{l}\right)=b_{L}\left(v_{L}^{l}\right)=0$ where $\min \left[v_{H}^{l}-\underline{v}_{H}, v_{L}^{l}-\underline{v}_{L}\right]=0$.

There exists a unique solution of the system of differential equations (3) satisfying the boundary conditions. To see this, consider an arbitrary ending point $\bar{x}^{\prime}$; given the ending point the system (3) determines unique paths $b_{i}(v), i=H, L$. Then either $b_{i}\left(\underline{v}_{i}\right) \geq 0$ as in path $i$ in the figure, or $b_{i}\left(v_{i}\right)=0$ at $v_{i}>\underline{v}_{i}$ as illustrated by path $i i$.

\section{Figure}

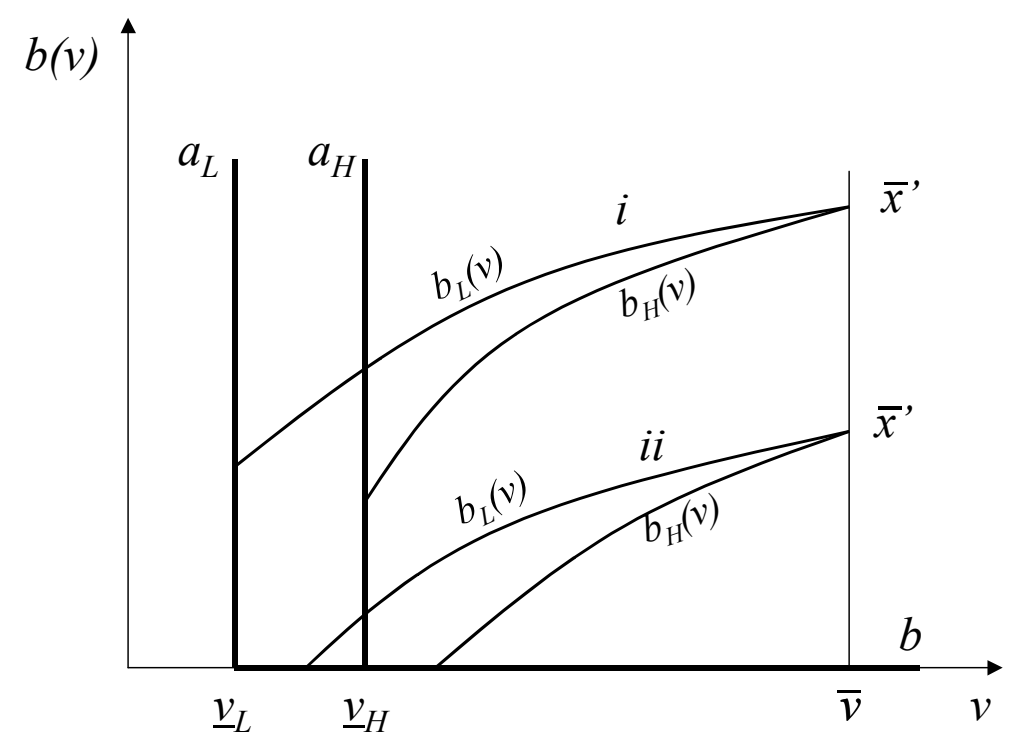


It follows from the real theory of differential equations that the points at which the $b_{i}(v)$ paths $(i=H, L)$ crosses the respective heavy lines $a_{i}-b$ are continuous functions of the chosen ending point $\bar{x}^{\prime}$. Furthermore, choosing a $\bar{x}^{\prime}$ close to zero certainly gives $b$-paths where $b_{i}(v)=0$ at $v_{i}>\underline{v}_{i}, \mathrm{i}=\mathrm{H}, \mathrm{L}$. Then if we can show that $\min \left[b_{H}\left(\underline{v}_{H}\right), b_{L}\left(\underline{v}_{L}\right)\right] \geq 0$ at a sufficiently large $\bar{x}^{\prime}$, it follows by continuity that at least one $\bar{x}$ exists such that $\min \left[b_{H}\left(\underline{v}_{H}\right), b_{L}\left(\underline{v}_{L}\right)\right]=0$. Due to the assumption that $c_{x}(x, v)>\varepsilon>0$, we know that

$$
\int_{\underline{v}_{L}}^{\bar{v}}\left[\frac{f_{L}(v) m}{c_{x}\left(b_{L}(v), e^{-1}(v)\right)}\right] d v \leq \frac{m}{\varepsilon} \text { and } \int_{\underline{v}_{H}}^{\bar{v}}\left[\frac{f_{H}(v) m}{c_{x}\left(b_{H}(v), e(v)\right)}\right] d v \leq \frac{m}{\varepsilon}
$$

Hence choosing $\bar{x}^{\prime}>m / \varepsilon$ yields $\min \left[b_{H}\left(\underline{v}_{H}\right), b_{L}\left(\underline{v}_{L}\right)\right] \geq 0$, and a solution exists.

It remains to be shown that the solution is unique. With our assumptions on the cost function, the first order derivatives of the $K_{i}$-functions, $i=H, L$, in the system (3), are continuous in all variables. Hence the solution is unique. 


\section{References.}

Amann,E. and W.Leininger (1996), “Asymmetric All-Pay Auctions with Incomplete Information: The Two-Contestant Case", Games and Economic Behavior, 14(1), 1-18.

Baker, G.P., M.C. Jensen and K. J. Murphy (1988), “Compensation and Incentive:

Practice vs. Theory", Journal of Finance, 43(3), 593-616.

Bhattacharya, S. and J. L. Guasch (1988), "Heterogeneity, Tournaments, and Hierarchies", Journal of Political Economy, 96(4), 867-881.

Che, Y-K and I. Gale (2003), "Optimal Design of Research Contests", American Economic Review, 93 (3), 646-671.

Clark, D. J. and C. Riis (2001), "Rank Order Tournaments and Selection”, Journal of Economics, 73 (2), 167-191.

Dasgupta, P. and E.Maskin (1998),"Efficient Auctions", Harvard Institute of Economic

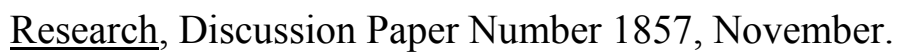

Fullerton, R.L. (1995), “Tournaments and Competition in Defense Acquisition Reform”, Doctoral Dissertation, University of Texas, Austin, Texas.

Fullerton, R.L., B.G. Linster, M. McKee and S. Slate (2002), "Using Auctions to Reward Tournament Winners: Theory and Experimental Investigations", The RAND Journal of Economics, 33 (1), 62-84.

Fullerton, R.L and R.P McAfee, (1999), “Auctioning Entry into Tournaments”, Journal of Political Economy, 107, 573-605.

Gifford, A. and R.W. Kenney, (1986), "The Production of Information through Labor Market Contests", Journal of Law, Economics and Organization, 2 (2), 305-313.

Green, J.R. and N.L. Stokey (1983), “A Comparison of Tournaments and Contracts”, Journal of Political Economy, 91 (3), 349-64.

Hvide, H.K. and E.G. Kristiansen, (2003), "Risk Taking in Selection Contests", Games and Economic Behavior, 42, 172-179. 
Judd, K.L., K. Schmedders and S. Yeltekin (2003), “ Optimal Rules for Patent Races”, mimeo, October.

Keck, O. (1988), “A Theory of White Elephants: Asymmetric Information in Government Support for Technology”, Research Policy, 17, 187-201.

Landers, R.M., J.B.Rebitzer and L.J.Taylor (1996), "Rat Race Redux: Adverse Selection in the Determination of Work Hours in Law Firms", American Economic Review, 86 (3), 329-348.

Lazear,E.P. and S.Rosen (1981), "Rank-Order Tournaments as Optimum Labor Contracts", Journal of Political Economy, 89 (5), 841-864.

Lazear, E.P. (1997),'Incentives in Basic Research”, Journal of Labor Economics, 15 (1), 167-197.

Liston-Heyes, C. (2001), "Setting the Stakes in Environmental Contests", Journal of Environmental Economics and Management, 41, 1-12.

McLaughlin, K.J, “Aspects of Tournament Models: A Survey”, Research in Labor Economics, 9, 225-256.

Meyer, M.A. (1991), “Learning form Coarse Information: Biased Contests and Career Profiles", Review of Economic Studies, 58, 15-41.

Meyer, M.A. (1992), "Biased Contests and Moral Hazard: Implications for Career Profiles" Annales D'Economie et de Statistique, 25/26, 165-187.

Moldovanu, B. and A. Sela, (2002), "Contest Architecture”, mimeo, January.

Nalebuff, B.J. and J.E. Stiglitz, (1983), "Prizes and Incentives: Towards a General Theory of Compensation and Competition”, Bell Journal of Economics, 14 (1), 21-43.

Riis, C. (2003), "Selection Efficiency in Contests", mimeo.

Singh, N. and D. Wittman, (2001), "Contests where there is Variation in the Marginal Productivity of Effort", Economic Theory, 18, 711-744.

Taylor, C.R. (1995), "Digging for Golden Carrots", American Economic Review, 85, 872890. 
Windus, M.L and D.D. Schiffel (1976), "Recoupment of Government R\&D

Expenditures: Issues and Practices in the USA”, Research Policy, 5, 180-196.

Yun, J. (1997), "On the Efficiency of the Rank-Order Contract under Moral Hazard and Adverse Selection”, Journal of Labor Economics, 15 (3), 466-494. 